(12) United States Patent Ewert et al.
(10) Patent No.:

(45) Date of Patent:
US 6,253,563 B1

Jul. 3, 2001

\section{(54) SOLAR-POWERED REFRIGERATION SYSTEM}

(75) Inventors: Michael K. Ewert, Seabrook: David J. Bergeron, III, League City, both of TX (US)

(73) Assignee: The United States of America as represented by the Administrator of the National Aeronautics and Space Administration, Washington, DC (US)

(*) Notice: Subject to any disclaimer, the term of this patent is extended or adjusted under 35 U.S.C. 154 (b) by 0 days.

(21) Appl. No.: 09/337,208

(22) Filed: Jun. 3, 1999

(51) Int. Cl. ${ }^{7}$ F25B 27/00

(52) U.S. Cl. 62/235.1; 62/228.3;62/201

$62 / 185$

(58) Field of Search …........................ 62/235.1, 228.1, $62 / 228.5,185,201,435,437,434 ; 165 / 10$

901.902

\section{References Cited}

\section{U.S. PATENT DOCUMENTS}

\begin{tabular}{|c|c|c|}
\hline & & \\
\hline 928 & * $5 / 1990$ & Gilbertson et al. \\
\hline 979 & $7 / 1990$ & it al \\
\hline & * 12/1994 & $62 \%$ \\
\hline 629 & $3 / 1996$ & Rafalovich \\
\hline - & $3 / 1996$ & 62 \\
\hline 600,218 & $* 10 / 1996$ & Jang \\
\hline & 97 & ........ \\
\hline & & \\
\hline
\end{tabular}

\begin{tabular}{|c|c|c|}
\hline & $3 / 1998$ & $318 /$ \\
\hline & & t al. \\
\hline & $5 /$ & ................... \\
\hline & $5 / 1$ & \\
\hline
\end{tabular}

* cited by examiner

Primary Examiner--Harry B. Tanner

(74) Attomey, Agent, or Firm-James M. Cate

\section{ABSTRACT}

A solar powered vapor compression refrigeration system is made practicable with thermal storage and novel control techniques. In one embodiment, the refrigeration system includes a photovoltaic panel, a variable speed compressor, an insulated enclosure, and a thermal reservoir. The photo voltaic (PV) panel converts sunlight into DC (direct current) electrical power. The DC electrical power drives a compressor that circulates refrigerant through a vapor compression refrigeration loop to extract heat from the insulated enclosure. The thermal reservoir is situated inside the insulated enclosure and includes a phase change material. As heat is extracted from the insulated enclosure, the phase change material is frozen, and thereafter is able to act as a heat sink to maintain the temperature of the insulated enclosure in the absence of sunlight. The conversion of solar power into stored thermal energy is optimized by a compressor control method that effectively maximizes the compressor's usage of available energy. A capacitor is provided to smooth the power voltage and to provide additional current during compressor start-up. A controller monitors the rate of change of the smoothed power voltage to determine if the compressor is operating helow or above the available power maximum, and adjusts the compressor speed accordingly. In this manner, the compressor operation is adjusted to convert substantially all available solar power into stored thermal energy.

20 Claims, 4 Drawing Sheets

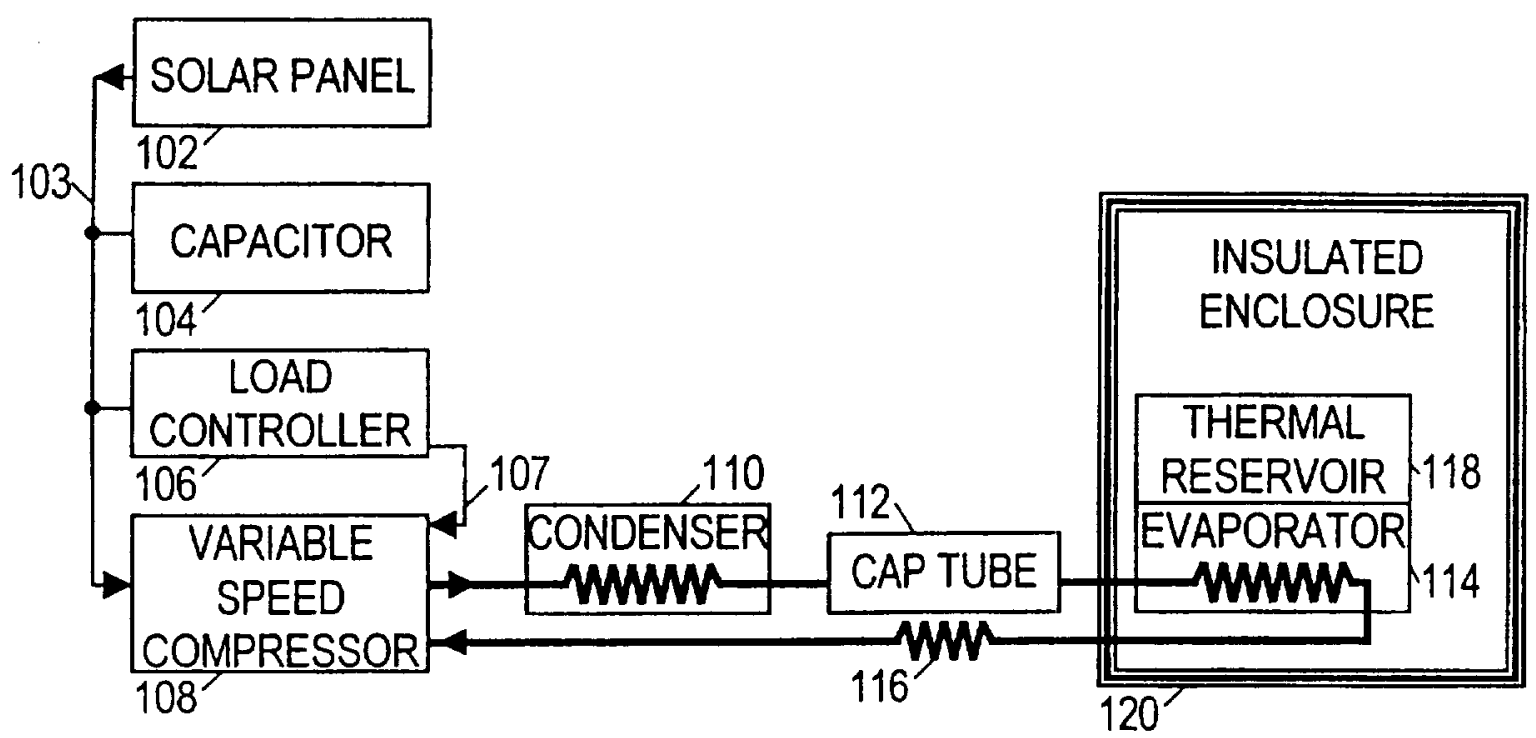




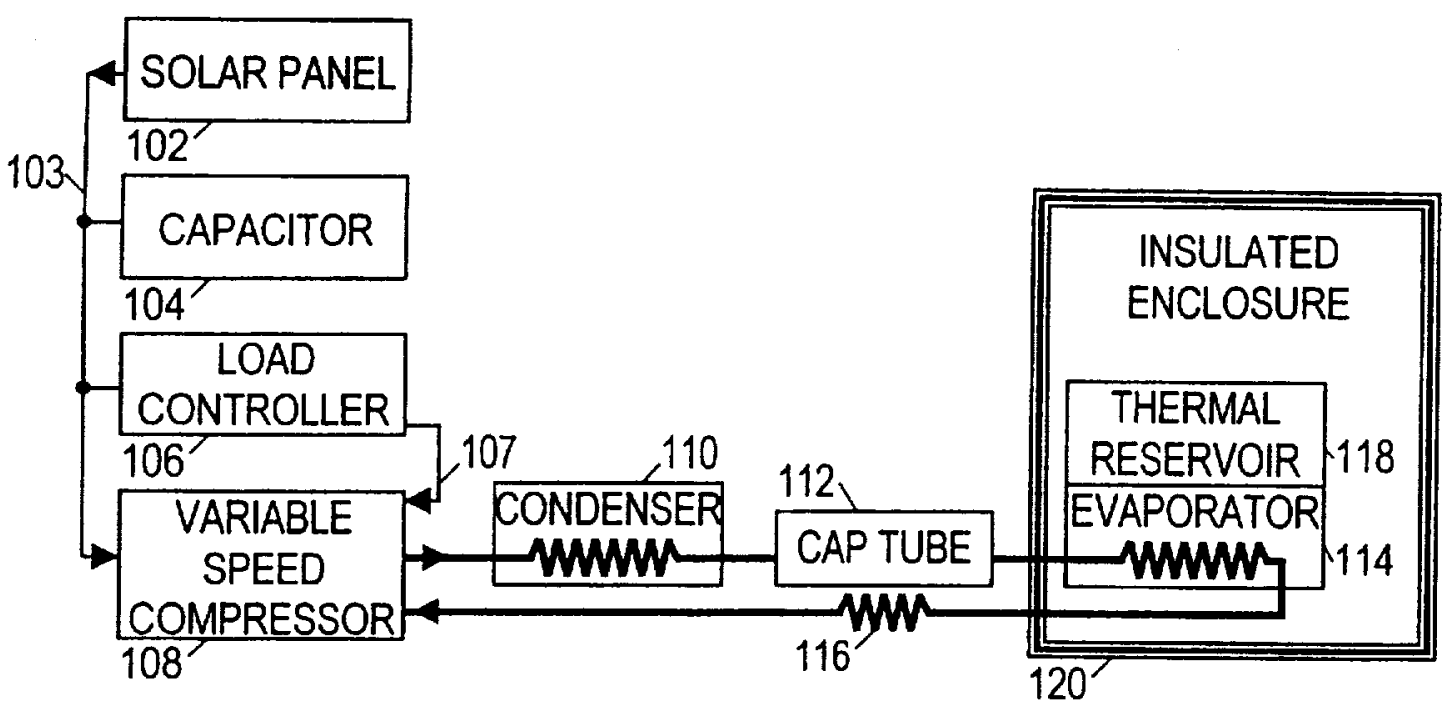

FIG. 1

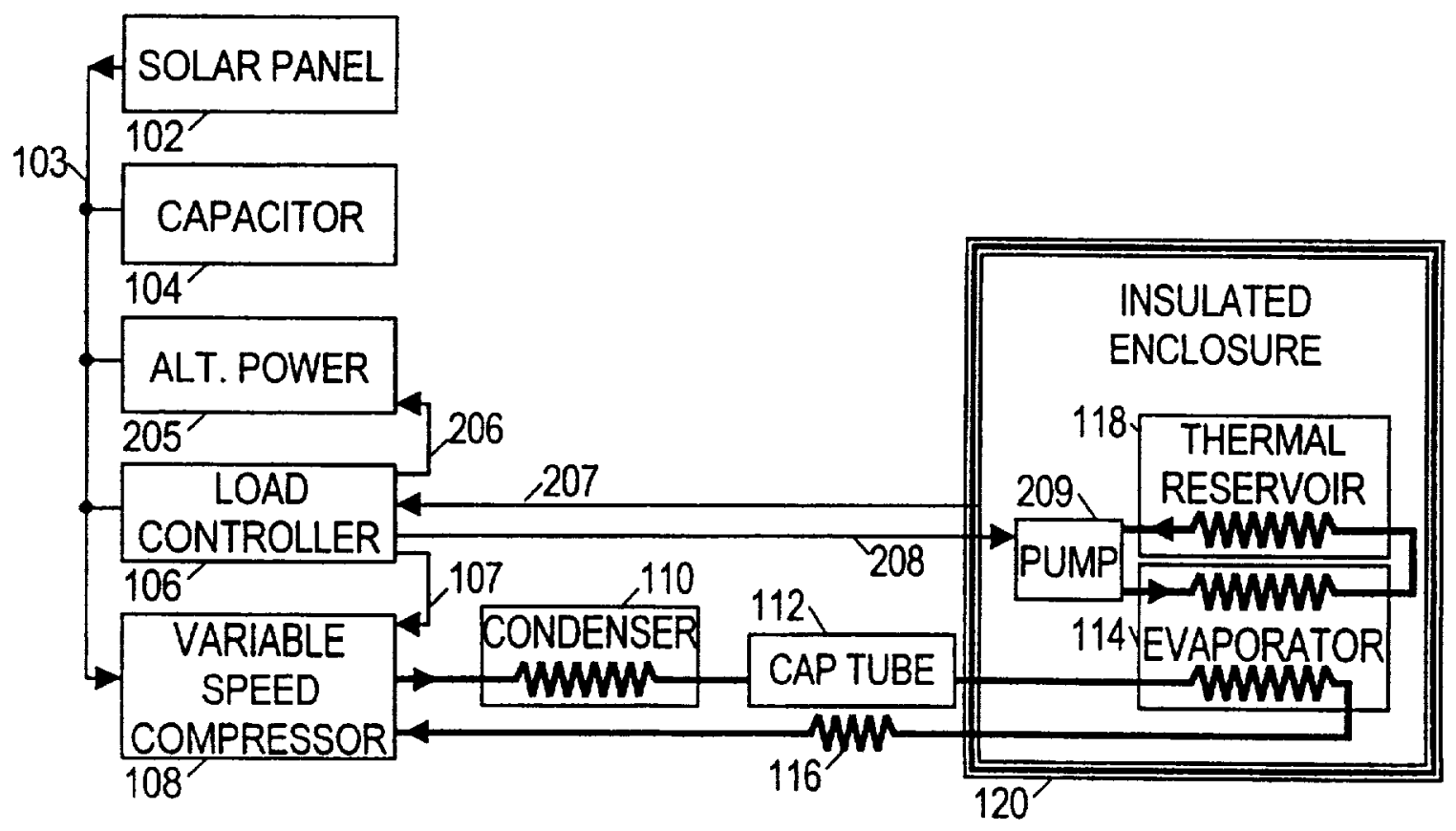

FIG. 2 


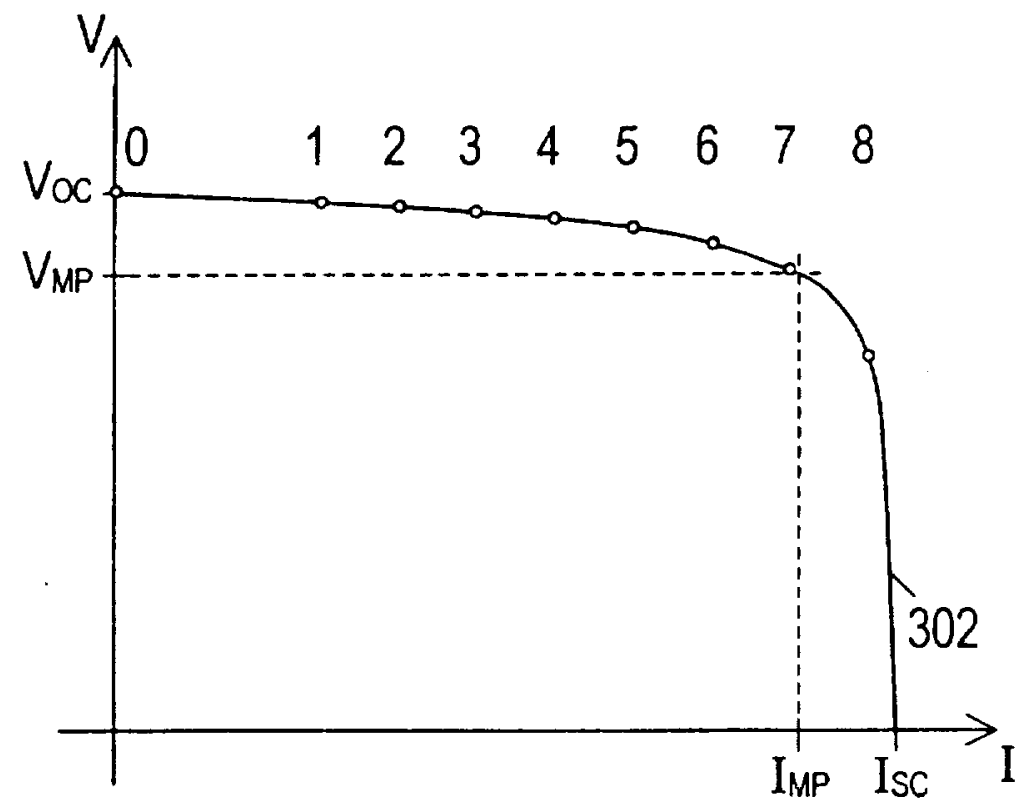

FIG. 3

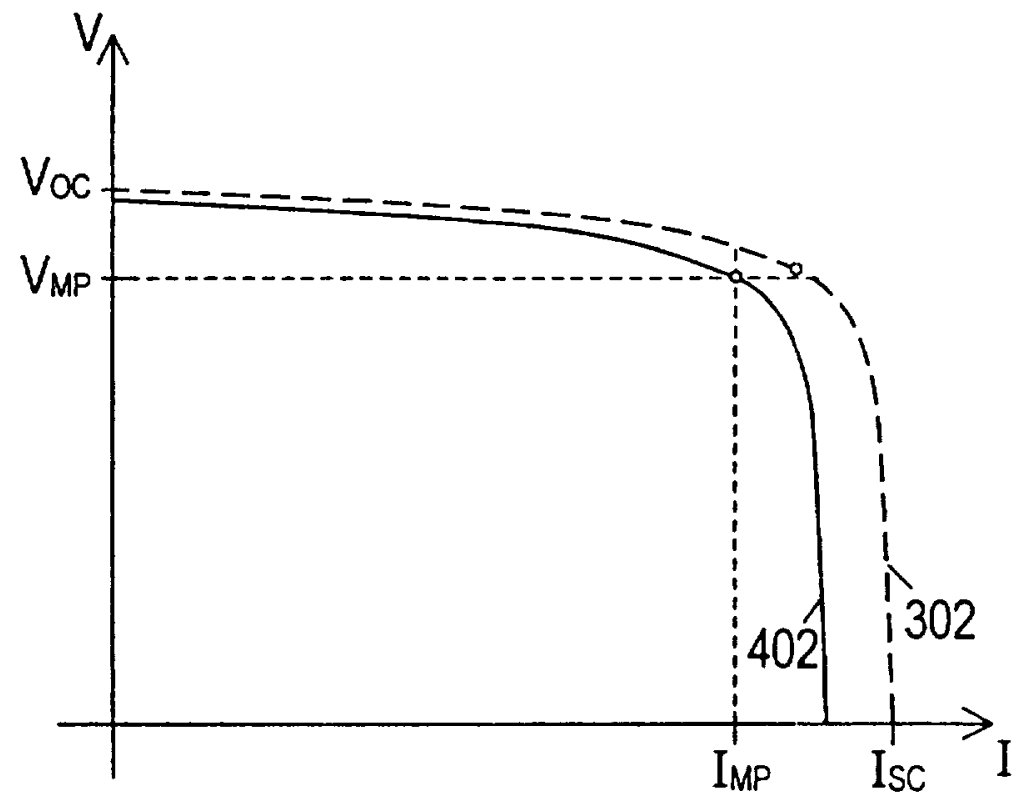

FIG. 4 


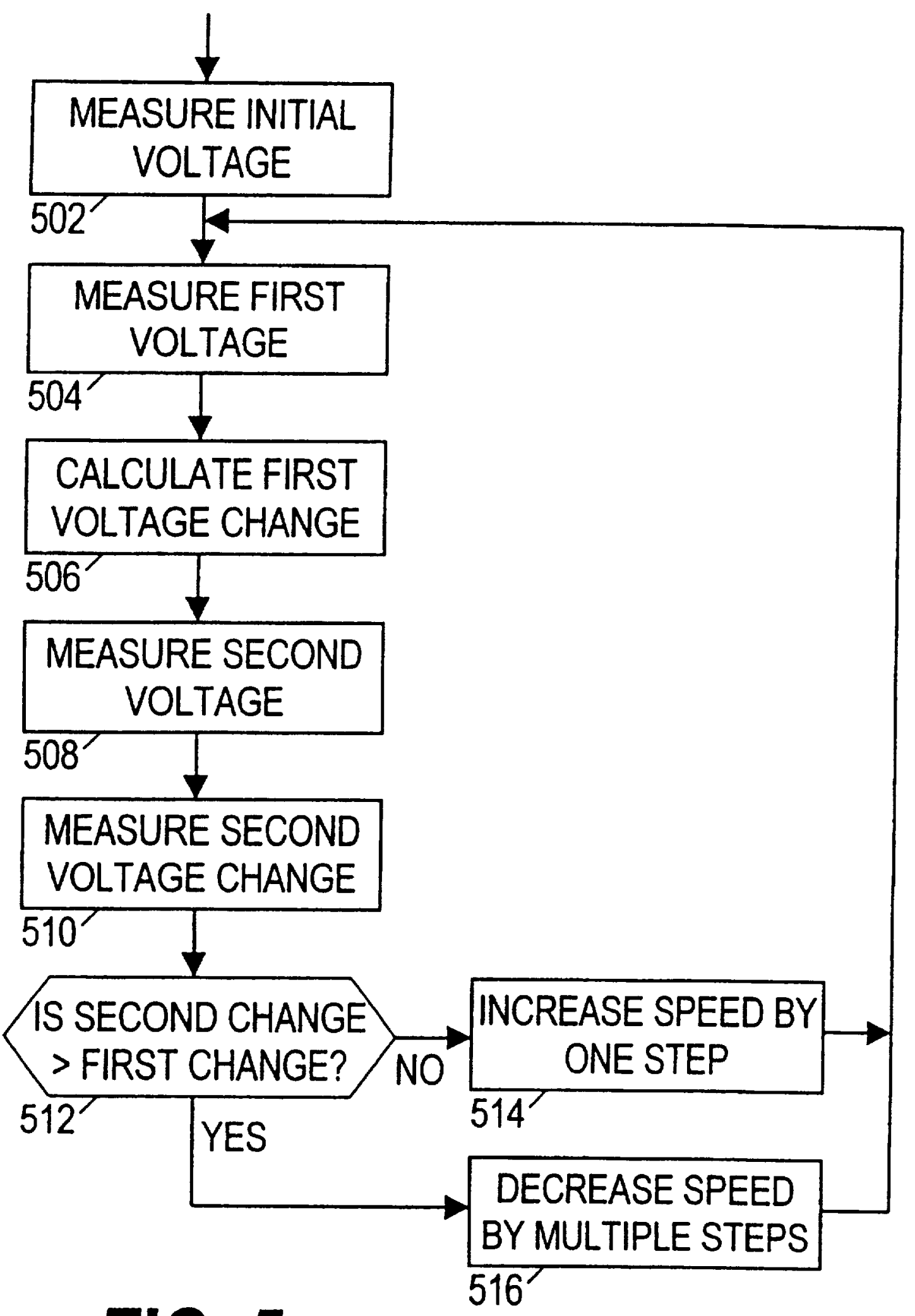

FIG. 5 
U.S. Patent Jul. 3, $2001 \quad$ Sheet 4 of $4 \quad$ US 6,253,563 B1

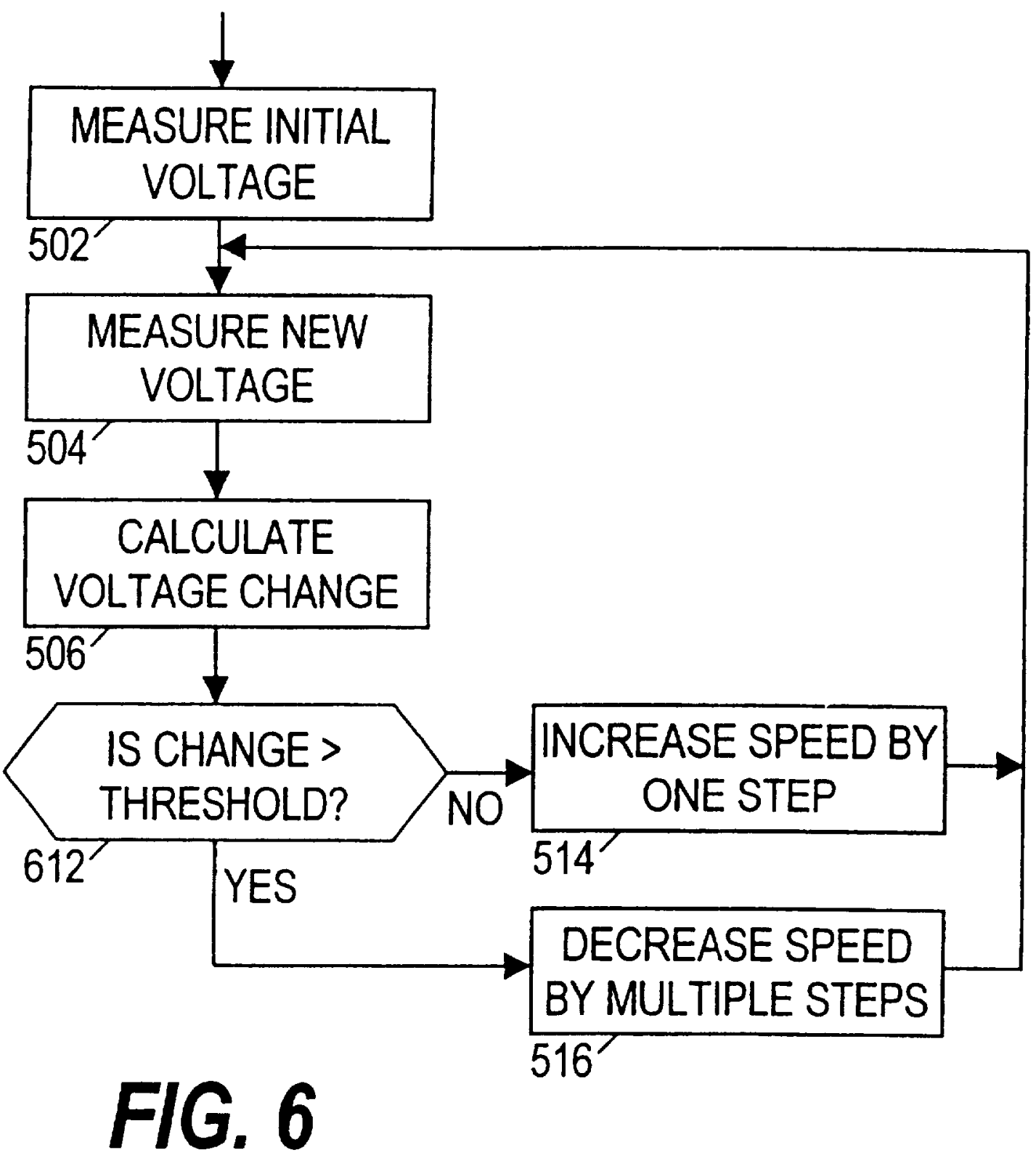




\section{1 SOLAR-POWERED REFRIGERATION SYSTEM}

\section{ORIGIN OF THE INVENTION}

The invention described herein was made in the performance of work under a NASA contract and is subject to Public Law 96-517 (35 U.S.C. $\$ 200$ et sq.). The contractor has not elected to retain title to the invention.

\section{BACKGROUND OF THE INVENTION}

1. Field of the Invention

This invention relates generally to solar power control systems, and in particular, to an efficient system and method for applying solar-generated power to refrigeration.

2. Description of the Related Art

Two billion people live without electricity. They represent a market for various solar powered systems such as standalone power systems and small capacity solar refrigerators. Efforts have been made to develop stand-alone photo voltaic (PV) power systems that provide lighting and power for small devices such as radios and small televisions. For example, such systems may include a solar panel, a battery. and a low wattage fluorescent light. Solar refrigerators, however, represent a bigger challenge.

Previous attempts to produce a marketable solar refrigerator have been largely unsuccessful. For example, consider the following patents:

In U.S. Pat. No. 4,126,014, Thomas Kay discloses an absorption refrigeration system powered by a heated fluid from a solar panel.

In U.S. Pat. No. 5,501,083, Tae Kim discloses an AC-powered air conditioner having a solar panel for backup electrical power.

In U.S. Pat. No. 5,497,629, Alexander Rafalovich discloses the use of solar power in an air conditioning system to pump heat from an indoor space to a thermal store.

In U.S. Pat. No. 5,685,152, Jeffrey Sterling discloses using a heated medium from solar collectors to produce a cold thermal store and mechanical energy to pump heat from an indoor space to the cold thermal store.

Kay's refrigeration system provides no means to maintain refrigerator operation in the absence of sunlight (e.g. at nighttime or on overcast days). As the air conditioning systems are largely unsuited for even small capacity refrigerators or freezers, no attempt has been made to scale these systems to produce a commercializable solar refrigerator.

Accordingly, it is desirable to provide an efficient, inexpensive, commercializable small capacity solar refrigerator which can operate for several days in the absence of sunlight. As batteries are often expensive and require regular maintenance, it would further be desirable to provide such a solar refrigerator which does not require batteries.

\section{SUMMARY OF THE INVENTION}

A solar powered vapor compression refrigeration system is made practicable with thermal storage and novel control techniques. In one embodiment, the refrigeration system includes a photovoltaic panel, a capacitor, a compressor, an insulated enclosure, and a thermal reservoir. The photovoltaic (PV) panel converts sunlight into DC (direct current) electrical power, some of which is stored in the capacitor. The capacitor provides additional current during compressor start-up, and thereafter acts to smooth out variations in the power voltage. The power from the PVT. Panel and capacitor drives the compressor to circulate refrigerant through a

\section{2}

vapor compression refrigeration loop, thereby extracting heat from the insulated enclosure. The thermal reservoir is situated inside the insulated enclosure and includes a phase change material. As heat is extracted from the insulated 5 enclosure, the phase change material is frozen. Thereafter the thermal reservoir is able to act as a heat sink to maintain the temperature of the insulated enclosure for an extended period in the absence of sunlight.

This conversion of solar power into stored thermal energy 10 is optimized by a compressor control method that effectively maximizes the compressor's usage of available energy. A controller monitors the rate of change of the smoothed power voltage to determine if the compressor is operating below or above the maximum available power, and adjusts

15 the compressor speed accordingly. In this manner, the compressor operation is continuously adjusted to convert substantially all available solar power into stored thermal energy.

\section{BRIEF DESCRIPTION OF THE DRAWINGS}

$A$ better understanding of the present invention can be obtained when the following detailed description of preferred embodiments is considered in conjunction with the following drawings, in which:

25 FIG. 1 is a block diagram of a first solar refrigeration system embodiment:

FIG. 2 is a block diagram of a second solar refrigeration system embodiment;

FIG. 3 is a graph of an exemplary I-V curve for a photovoltaic panel;

FIG. 4 is a graph of an exemplary I-V curve for a photovoltaic panel in reduced light;

FIG. 5 is a flowchart of a first compressor speed control 35 method: and

FIG. 6 is a flowchart of a second compressor speed control method.

Whle the invention is susceptible to various modifications and alternative forms, specific embodiments thereof are 40 shown by way of example in the drawings and will herein be described in detail. It should be understood, however, that the drawings and detailed description thereto are not intended to limit the invention to the particular form disclosed, but on the contrary, the intention is to cover all 45 modifications, equivalents and alternatives falling within the spirit and scope of the present invention as defined by the appended claims.

\section{DETAILED DESCRIPTION OF PREFERRED EMBODIMENTS}

Turning now to the figures, FIG. 1 shows a first embodiment of a solar refrigeration system which includes a solar panel 102 connected to a power bus 103 . Although a wide variety of solar panel types and styles may be employed, one 55 suitable example is a 12 volt nominal PV panel that is capable of a peak power output of approximately 120 watts at approximately 15 volts under full solar insolation.

A capacitor 104 is connected to power bus 103 in parallel with solar panel 102. Capacitor 104 operates to provide 60 temporary storage of electrical charge in order to smooth any voltage variations on power bus $\mathbf{1 0 3}$ and to provide extra current during demand periods. The voltage variations may be caused by a variety of sources including changes in light intensity on the solar panel and changes in the electrical load 65 driven by the solar panel 102 . The capacitor 104 may be varied in size and type, but a preferred example is a 0.2 Farad electrolytic capacitor. 
3

A variable speed compressor 108 with a load controller 106 is directly coupled to the solar panel 102 by power bus 103. In this context, "directly coupled" is defined to mean that no power converters are provided between the compressor 108 and solar panel 102. Although other embodiments are also contemplated, this embodiment advantageously exhibits relatively high efficiency due to the direct powering of the compressor $\mathbf{1 0 8}$ by a PV panel. It is noted that systems which use batteries typically force the solar panel to operate below its peak power point to match the battery charging voltage. Powering the compressor directly from the solar panel allows the solar panel to be operated at the maximum power point.

The variable speed compressor $\mathbf{1 0 8}$ is preferably a direct current compressor such as a Danfoss® BD35F direct current compressor with refrigerant 134a. Persons of skill in the art will recognize that other suitable compressors and refrigerants can be employed. The BD35F includes a "brushless" DC (direct current) motor in that provides permanent magnets on the rotor. Electronics in the BD35F switch the DC input to provide a 3 -phase input to fixed coils that drive the rotor. The electronics improve the motor's efficiency by sensing the back-EMF in the coils to determine the rotor position. This compressor implementation is believed to exhibit efficiency and longevity advantages over typical DC compressors. As discussed in further detail below, load controller 106 senses the voltage on power bus 103 and regulates the speed of compressor 108 in response to variations in this voltage.

Compressor 108 circulates refrigerant through a vapor compression refrigeration loop that preferably includes a first heat exchanger (a.k.a. a condenser) 110, a capillary tube 112. a second heat exchanger (a.k.a an evaporator) 114 internal to an insulated enclosure 120, and a third heat exchanger (sometimes referred to as SLLL HX, or the suction line/liquid line heat exchanger) 116 associated with the capillary tube 112. As refrigerant is circulated through the loop, it is compressed by compressor 108 , cooled to a liquid state by ambient air in condenser 110, flash-cooled by heat exchanger 116 in capillary tube 112, evaporated to a gaseous state in evaporator 114, warmed by heat exchanger 116, and recompressed and re-circulated by compressor 108 . This circulation results in a net transfer of heat from the evaporator 114 to the condenser 110 , thereby cooling the interior of the insulated enclosure 120 by heating ambient air. One of skill in the art will readily recognize that this refrigerant loop may be constructed in various suitable manners, and that other refrigerant loops may also be employed to achieve a net transfer of heat energy away from the insulated enclosure 120 without departing from the scope of the invention. For example, one specific alternate implementation uses an expansion valve in place of the capillary tube 112.

Similarly, many types of insulated enclosures are well known and may be employed, but a preferred construction for the insulated enclosure 120 uses fiberglass-reinforced plastic shells for the cabinet with vacuum panels between the inner and outer shells for insulation. A bezel interface is preferably provided between the cabinet and the door to minimize thermal conductance and convection through the seal. With this preferred construction, a cabinet having a composite $\mathrm{R}$ value (thermal resistance in units of $\mathrm{hr} \cdot \mathrm{ft}^{2} \cdot{ }^{\circ} \mathrm{F} . /$ BTU) of 26 has been achieved. (Most conventional refrigerators have a composite $R$ value of 5 .)

Referring still to FIG. 1, the load controller 106 senses the 6 voltage on power bus 103 and provides a speed control signal 107 to variable speed compressor 108 . By controlling
4

the compressor speed, the load controller 106 effectively maximizes the power extracted from the solar panel. It inexpensively implements an advantageous optimization method as described in further detail below. While it can 5 take various forms, the load controller 106 is preferably implemented in the form of a microcontroller that implements a software algorithm. The microcontroller may also be designed to perform other system functions such as: monitoring internal temperature of the insulated enclosure, monitoring the compressor for error conditions, and initiating compressor start-ups and shut-downs in a manner designed to extend the life of the compressor. In alternate embodiments, the load controller 106 may also control power source switching to access alternate power sources, if available and when necessary, or to provide redundancy (in the case of multiple solar panels).

A thermal reservoir 118 is preferably provided in the insulated enclosure 120 . Thermal reservoir 118 preferably comprises a phase-change material that has a phase-change 20 temperature at or slightly below the target interior temperature for the insulated enclosure. Particularly desirable phasechange materials are those having a solid-liquid phase change with a high heat of fusion, and which are inexpensive and relatively non-toxic. Water and water solutions are 5 examples of suitable phase change materials. A water solution of approximately 3-5\% propylene glycol may be particularly desirable, as it exhibits a reduced tendency to rupture closed containers when freezing. The size and phase change material of the thermal reservoir is preferably chosen to maintain the target interior temperature for several days in the absence of solar power (or at least 36 hours). One of skill in the art will recognize that thermal reservoir 118 may be implemented in a variety of suitable configurations.

In the embodiment of FIG. 1, the thermal reservoir 118 is 35 contemplated as being adjacent to evaporator 114, and/or as being a part of evaporator 114. As refrigerant circulates through the evaporator 114 to cool the interior of the insulated enclosure 120, a direct transfer of heat energy occurs to evaporatci 114 from thermal reservoir 118 to cool 40 the thermal reservoir and induce a phase change of the phase-change material. In other words, if the phase-change material is water, the flow of refrigerant through the evaporator cools and freezes the water.

In operation, the solar panel $\mathbf{1 0 2}$ delivers power to power 45 bus 103 during the day when the sun is shining. The load controller 106 runs the compressor 108 at a speed that maximizes the power extracted from the solar panel. The compressor 108 circulates refrigerant through a refrigerant loop to cool the insulated enclosure and to cool and induce so a phase change of the material in the thermal reservoir. At night and during adverse weather conditions, no power is delivered to the power bus 103 , and the compressor 108 is inactive. The temperature in the insulated enclosure is maintained by the thawing of the material in the thermal 55 reservoir. Advantageously, no fluid circulation or active heat pumping is required to maintain the enclosure temperature during these inactive time periods.

Referring now to FIG. 2, a second solar refrigeration system embodiment is shown. In this embodiment, an alter60 nate power source 205 is coupled to power bus 103 . The alternate power source $\mathbf{2 0 5}$ may take many forms including, e.g. a supplemental battery, a fuel cell, a generator, or an AC/DC converter connected to a commercial AC power grid. The load controller 106 turns the alternate power source 205 on or off by means of an enable signal 206 . The load controller 106 preferably minimizes the use of alternate power source 206 to the greatest extent possible, using it 
US $6,253,563 \mathrm{~B} 1$

5

only when solar power is unavailable and the temperature of the insulated enclosure exceeds a predetermined threshold. The load controller 106 monitors the interior temperature of insulated enclosure $\mathbf{1 2 0}$ by means of a temperature signal 207 from a temperature sensor (not shown) in insulated enclosure 120.

The solar refrigeration system embodiment of FIG. 2 also employs an alternate configuration for the evaporator 114 and thermal reservoir 118. In this configuration, the refrigerant passing through evaporator 114 cools a second fluid that is pumped through the evaporator 114 by a pump 209. Many fluids may be used, but currently a propylene glycol and water mixture is preferred. The cooled second fluid is then circulated through a heat exchanger in the thermal reservoir 118 to cool and induce a phase change in the phase change material. The load controller 106 may be configured to turn pump 209 on and off by means of a signal 208. Pump 209 is preferably activated only when compressor 108 is operating. A fan may be provided to improve air circulation, and may also be controlled by signal 208.

In one particular implementation of the alternate configuration shown by FIG. 2, the cooling of the insulated enclosure 120 is accomplished primarily by the thermal reservoir 118 and the heat exchanger therein. This implementation may prove advantageous relative to the configuration shown in FIG. 1 for several reasons. A first feature of this implementation is that the refrigerant volume is reduced, which may provide reduced cost and increased system longevity. A second feature of this implementation is that thermal leakage to the interior of the insulated enclosure during and after compressor shut-down is reduced. A third feature is that mechanical design of the thermal reservoir may be simplified due to a larger and more favorably distributed heat exchange area with the phase change material. It is noted that the solar refrigeration system embodiment of FIG. 1 may be modified to use this thermal reservoir configuration.

The load controller 106 may be designed to monitor the temperature of the insulated enclosure and respond to temperature excursions above or below predetermined thresholds. As mentioned previously, the load controller 106 may activate alternate power source $\mathbf{2 0 5}$ in response to a detected temperature above an upper temperature limit. Also, the load controller 106 may halt the variable speed compressor 108 in response to a detected temperature below a lower temperature limit. Once the temperature returns to the desired range, the load controller 106 may then resume normal solar-powered operation. One of skill in the art will recognize the desirability of providing some hysteresis in any such temperature regulation strategy. It is noted that the upper temperature limit is preferably slightly above the phase change temperature, and the lower temperature limit is preferably slightly below the phase change temperature.

As previously mentioned, load controller 106 operates to maximize the power drawn from the solar panel 102. Various methods which may be implemented by the load controller are now described with reference to FIGS. 3 and 4. FIG. 3 shows an I-V curve $\mathbf{3 0 2}$ representing the voltage V provided by solar panel 102 as a function of current I drawn from the solar panel, assuming maximum insolation (sunlight intensity). The voltage varies from $\mathrm{V}_{o c}$ when no current is drawn to 0 when the short circuit current $I_{S C}$ is drawn. A typical example of an open circuit voltage $V_{o C}$ for a nominal 12 volt panel is 20 volts, and a typical example of a short circuit current is 8 amperes. On the curve between these two points is a maximum power point $\left(\mathrm{I}_{M P}, V_{M P}\right)$ where the maximum power is extracted from the solar panel. This point occurs where the slope of the curve is $\mathrm{dV} / \mathrm{dl}=-$ V/I.

\section{6}

The load controller 106 preferably locates this maximum power point by an iterative search process. At an initial time $\mathrm{t}=0$, the compressor $\mathbf{1 0 8}$ is not running, and no current is drawn. The load controller determines that a sufficient 5 start-up voltage exists and starts the compressor at a minimum startup speed. Note that the current drawn by the compressor increases as the speed of the compressor increases. At a subsequent time $t=1$, the compressor is drawing a current and the voltage provided by the solar panel has been slightly reduced. The load controller 106 then begins gradually increasing the speed of the compressor 108. detecting the power bus voltage at regular intervals and adjusting the speed of the compressor in response to some criterion based on the detected voltage. The time progression is of operating points has been exaggerated for illustration. In a preferred embodiment, the increments in speed are digital and are much smaller, so that 255 or more operating points on the curve are possible.

Various adjustment criteria may be used. For example, referring momentarily to FIG. $\mathbf{4}$, a second I-V curve $\mathbf{4 0 2}$ is shown for reduced insolation. The maximum power point on curve $\mathbf{4 0 2}$ has shifted relative to the maximum power point on curve 302. It is noted that while the current $I_{M P}$ at the maximum power point is particularly sensitive to the amount 5 of insolation, the voltage $V_{M P}$ at the maximum power point is relatively insensitive to the amount of insolation. Consequently, the load controller 106 may increase or decrease the compressor speed as needed to maintain the power bus voltage close to a predetermined voltage target, 30 e.g. the maximum power voltage for full solar insolation.

While simple, this criterion is suboptimal since the maximum power voltage varies with temperature, and in any case, this criterion does not provide for full power extraction during reduced insolation. Referring again to FIG. 3, it is 5 noted that at all operating point voltages on curve 302 above the maximum power point voltage, the power provided by the solar panel increases as the current increases, whereas for all operating point voltages on the curve below the maximum power point voltage, the power provided by the 40 solar panel DECREASES as the current increases. When this observation is combined with the observation that the power required by the compressor always increases as the speed increases, an improved control method can be developed for the load controller 106.

Referring simultaneously to FIGS. 1 and 3, it is noted that when the compressor 108 is run at a speed requiring less power than the solar panel 102 can provide, an increase in compressor speed will result in a matching increase in power extracted from the solar panel. Due to the capacitor 104, the 50 power bus voltage will decrease smoothly and stabilize. In other words, the magnitude of the time derivative of the voltage decreases as a function of time. When the compressor 108 is run at a speed requiring more power than the solar panel 102 can provide, the charge on capacitor 104 provides 55 the extra power required. Since only a limited amount of charge exists on capacitor 104, the capacitor 104 is increasingly depleted as time goes on, and the compressor attempts to draw more current from solar panel 102. This in turn causes the solar panel to provide less power as the voltage 60 drops, causing further depletion of the capacitor and even more current draw from the solar panel 102. The power bus voltage rapidly decays, and in fact, the rate of voltage decay increases as a function of time. Expressed in calculus terms, when the second derivative of the voltage with respect to 65 time is greater than or equal to zero, the system is operating on the curve above the maximum power point voltage. When the second derivative of the voltage with respect to 


\section{US $6,253,563 \mathrm{~B} 1$}

7

time is less than zero, the system is operating on the curve below the maximum power point voltage.

FIG. 5 shows a first improved control method which may be implemented by load controller 106. After the load controller has started the compressor and allowed some small amount of time for the voltage on the power bus to settle into a steady state, the load controller begins sampling the voltage at regularly spaced time intervals. One of skill in the art will recognize that the sampling intervals may be allowed to vary if this is determined to be desirable, and appropriate adjustments can be made to the method Additionally, the power bus voltage signal may be mildly conditioned to remove high frequency noise before being sampled by the load controller.

In step $\mathbf{5 0 2}$ an initial voltage sample is taken before the load controller enters a loop consisting of steps 504-516. For each iteration of the loop, two additional voltage samples are taken. In step 504, a first voltage sample is taken, and in step 506 a first change in the voltage is calculated by subtracting the previous voltage sample from the first voltage sample. In step 508, a second voltage sample is taken, and in step $\mathbf{5 1 0}$ a second voltage change is calculated by subtracting the first voltage sample from the second voltage sample.

In step 512, the two calculated voltage changes are compared. If the magnitude of the second voltage change is less than or equal to the magnitude of the first voltage change, then in step 514. the loop controller increments the speed of the compressor by one step. On the other hand. if the magnitude of the second voltage change is larger than the magnitude of the second voltage change, then in step $\mathbf{5 1 6 .}$ the loop controller decrements the speed of the compressor by two or more steps. While various implementations of decrement step 516 are contemplated, it is currently preferred to make the number of decrement steps a predetermined constant based on the system embodiment. It is further contemplated to make the increment step sizes adaptive in nature. The adaptation may be based on the size of the calculated first voltage change, so that smaller voltage changes result in smaller step sizes. In this manner, the load 40 controller may more quickly and accurately locate the maximum power point. The nature of the adaptation may be changed after the first time the speed is decremented to provide for a smaller range of variation about the optimal operating point. For example, the step size may become 45 based proportionally on the size of the second calculated voltage change, so that larger voltage changes result in larger step sizes.

FIG. 6 shows a second improved control method which may be implemented by load controller 106. When the 50 system is operating on the portion of the solar panel curve below the maximum power point, the calculated voltage changes continually grow if the compressor speed is not adjusted. Hence the method of FIG. 5 may be simplified by eliminating steps 508 and 510, and replacing step 512 with step 612, in which the calculated voltage change is compared with a predetermined threshold. No matter where the system is operating on the lower part of the curve, eventually the calculated voltage change will exceed the threshold, and the compressor speed will be reduced accordingly. When the voltage change is less than the threshold, the system is assumed to be on the upper part of the curve, and the compressor speed is increased. The threshold is preferably adjusted to allow for only a small range of variation around the maximum power point.

It is noted that the disclosed refrigeration systems and power control methods may have many varied embodi-
8

ments. For example, one refrigeration system embodiment may employ an insulated enclosure with divided compartments that are maintained at different temperatures such as might be suitable for storing fresh and frozen foods. Another 5 embodiment may employ the structure and stored contents of the insulated enclosure as the thermal reservoir. This latter approach may prove particularly suitable for refrigeration systems that are configured to produce the stored contents. such as would be the case for an ice maker. Some embodi10 ments may include alternate energy sources such as batteries, a generator, or a commercial power grid, the use of which is may be minimized by using the solar panel as much as possible. These embodiments could use a smaller thermal reservoir due to avililability of an alternate power source to 15 maintain the temperature. In some embodiments, the refrigeration system may be applied to cool poorly insulated enclosures that are often exposed to substantial amounts of sunlight. In this vein. one refrigeration embodiment is an air conditioning system for vehicles that cools the interior when 20 the vehicle is exposed to the sun. Such a system may or may not include some form of phase change material as a thermal reservoir.

Numerous such variations and modifications will become apparent to those skilled in the art once the above disclosure 5 is fully appreciated. It is intended that the following claims be interpreted to embrace all such variations and modifications.

What is claimed is:

1. A refrigeration system which comprises:

an enclosure having an interior space;

phase-change material situated in said interior space;

a photovoltaic panel configured to provide electrical power having a DC voltage; and

a compressor electrically connected to the photovoltaic panel to receive electrical power at said DC voltage. wherein the compressor is configured to circulate refrigerant through a first heat exchanger to cool the refrigerant, through a constriction configured to sustain a pressure drop, and through a second heat exchanger to extract heat from said interior space.

2. The refrigeration system of claim 1 , further comprising:

a pump configured to circulate a fluid through the second heat exchanger to cool the fluid, and through a third heat exchanger to extract heat from the phase change material.

3. The refrigeration system of claim 1 , wherein at least a portion of the second heat exchanger is situated adjacent to the phase change material.

4. The refrigeration system of claim 1, wherein the phase-change material mass, when solidified, is sufficient to maintain the interior space of the enclosure substantially at a phase-change temperature for more than 36 hours.

5. The refrigeration system of claim 1 , wherein the 55 phase-change material comprises water.

6. The refrigeration system of claim 1 , further comprising a capacitor coupled to the compressor to smooth variations in said DC voltage

7. The refrigeration system of claim 6 , wherein the 60 compressor is a variable speed compressor, and wherein the refrigeration system further comprises a controller configured to monitor the $\mathrm{DC}$ voltage and to regulate the compressor speed to run the compressor at a substantially maximum available: power.

658 . The refrigeration system of claim 7 , wherein the controller is configured to receive a temperature signal indicative of a ternperature of the interior space of the 


\section{US $6,253,563 \mathrm{Bl}$}

9

enclosure, and wherein the controller is configured to halt the compressor if the temperature falls below a lower temperature limit.

9. The refrigeration system of claim 7 , further comprising an alternate energy source, wherein the controller is config- 5 ured to receive a temperature signal indicative of a temperature of the interior space of the enclosure, and wherein the controller is configured to enable the alternate energy source if the temperature rises above an upper temperature limit.

10. The refrigeration system of claim 7 , wherein the controller is configured to calculate a voltage rate-of-change magnitude, wherein the controller is configured to increment the compressor speed when the voltage rate-of-change magnitude is below a predetermined threshold, and wherein the controller is configured to decrement the compressor speed 1 when the voltage rate-of-change magnitude is above a predetermined threshold.

11. The refrigeration system of claim 1 , wherein the compressor has a direct electrical connection to the photovoltaic panel.

12. The refrigeration system of claim 1, wherein the compressor is a DC powered, variable speed compressor, the output of which is responsive to an amount of received solar radiation.

13. A solar powered refrigeration apparatus, comprising: 2 a compressor;

a solar powered electrical power source electrically connected to said compressor;

a thermal energy storage device having communication with said compressor; and

control means, associated with said power source, for accumulating electrical power derived from said power source and for conducting accumulated electrical power, and continuous electrical power derived from 35 said power source, to said compressor.

14. The solar powered refrigeration apparatus of claim 13, wherein the thermal energy storage device comprises a phase change material.

15. A solar powered refrigeration apparatus which comprises:
10

a compressor system;

a solar powered electrical power source electrically connected to said compressor system; and

a control means, associated with said solar power source, for accumulating electrical power derived from said solar power source, and for conducting accumulated electrical power and continuous power derived from said solar power source to said compressor system.

16. The apparatus of claim 15, wherein said control means 10 includes a capacitive power storage means.

17. The apparatus of claim 15, wherein said solar power source is directly electrically connected to said compressor system during start-up of said compressor system.

18. The apparatus of claim 15 , wherein said control means further comprises means for increasing a level of power applied to said compressor during start-up of said compressor by applying both continuous power and an increased level of accumulated power to said compressor.

19. The apparatus of claim 15, wherein said control means 20 comprises means for repeatedly adjusting, by means of motor speed adjustments, a load associated with said motor to enable said motor to use substantially all of the available power.

20. A power control system for efficiently applying power received from a solar collector array to an electric motor, wherein the system comprises:

buffer circuitry connected to receive power from said solar collector array, the buffer circuitry including means for accumulating electrical power derived from said solar collector array;

speed control means associated with said electric motor; and

control circuitry associated with said buffer circuitry and said electric motor for repetitively monitoring the motor load and available power available from both said solar collector array and said buffer circuitry, and for adjusting said speed control means to cause said motor to utilize substantially all of the available power. 